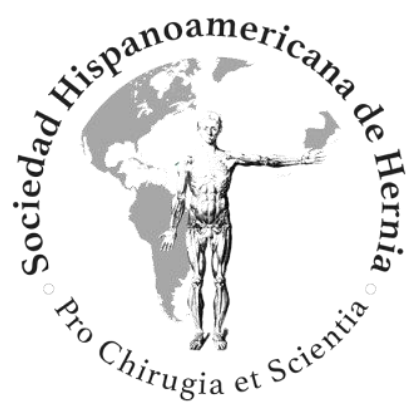

Eventración subcostal compleja: uso de saco y prótesis en la resolución

\title{
Complex subcostal eventration: use of sac and prosthesis in resolution
}




\section{Eventración subcostal compleja: uso de saco y prótesis en la resolución}

Complex subcostal eventration: use of sac and prosthesis in resolution

Andrés Felipe Escudero-Sepúlveda ${ }^{1}$, María Camila Otero-Rosales ${ }^{2}$, Fabián Leonardo Escudero Sepúlveda ${ }^{3}$

${ }^{1}$ Universidad de Buenos Aires. Servicio de Cirugía General, Laparoscópica y Miniinvasiva. Hospital Municipal de Trauma y Emergencias Dr. Federico Abete. Gran Buenos Aires (Argentina). 2Universidad Autónoma de Bucaramanga. Floridablanca, Santander (Colombia). ${ }^{3}$ IPS SURA. Rionegro, Antioquía (Colombia)

Autor para correspondencia: Andrés Felipe Escudero-Sepúlveda. Servicio de Cirugía General, Laparoscópica y Miniinvasiva. Hospital Municipal de Trauma y Emergencias Dr. Federico Abete. 123, Miraflores. Pablo Nogués. Provincia de Buenos Aires (Argentina) Correo electrónico: dr.andresescudero@gmail.com

\section{RESUMEN}

Las hernias incisionales son las complicaciones más frecuentes de la cirugía abdominal. Se originan por el cierre de una laparotomía. Se presenta el caso de una mujer con eventración compleja subcostal derecha con pérdida de domicilio. Se propone una técnica con el uso de sacos y una malla.

Palabras claves: Cirugía, hernia compleja, hernia subcostal, pérdida de domicilio, neumoperitoneo preoperatorio, separación de componentes anterior.

\section{ABSTRACT}

Incisional hernias are the most frequent complication of abdominal surgery, they are caused by the closure of a laparotomy, the case of a 
woman with complex right subcostal hernias with loss of home is presented, a technique is proposed with the use of sacks and a mesh.

Keywords: Surgery, complex hernia, subcostal hernia, loss of domian hernia, preoperative pneumoperitoneum, anterior component separation.

\section{INTRODUCCIÓN}

Las eventraciones son las complicaciones más frecuentes de la cirugía abdominal. Se originan por el cierre de una laparotomía ${ }^{1,2}$. Su incidencia está entre un 15 y un $20 \%$ de todas las laparotomías ${ }^{3}$. En Estados Unidos la tasa de incidencia está cerca del $20 \%$. Su reparación es exclusivamente quirúrgica² .

Las eventraciones pueden surgir a partir de las incisiones subcostales derechas descritas por Kocher (de quien recibieron su nombre), en la sigue un margen costal, lo que proporciona una buena presentación de la vía biliar ${ }^{4}$.

La eventración compleja es aquella que, por su localización, tamaño del defecto herniario, dimensiones del saco herniario o procesos locales agregados a la hernia representa una condición poco usual. Entre estas se encuentran los defectos herniarios próximos a márgenes óseos, los defectos gigantes y las hernias con pérdida de domicilio, entre otras ${ }^{5}$. La eventración complicada tiene altos índices de morbimortalidad y recidiva, y alcanza una cifra de entre el 11 y el $29 \%$. La recidiva en defectos mayores a $5 \mathrm{~cm}$ puede alcanzar una tasa del $43 \%^{6}$.

Se estima que entre un 2 y un $5 \%$ de las eventraciones son posteriores a una incisión subcostal de Kocher ${ }^{7}$.

Las eventraciones resultantes de las incisiones de Kocher (transversa de Ross, subcostal bilateral y transversal ampliada de West) condicionan la separación progresiva de los segmentos musculares de los rectos, con lo que su reparación resulta difícil, al quedar la sutura con una tensión excesiva cuando se consigue ${ }^{7}$. 


\section{CASO CLÍNICO}

Se presenta paciente femenina de 65 años, hipertensa, controlada en tratamiento con enalapril, con antecedentes de colecistectomía abierta por colecistitis aguda litiásica hace cinco años en otra institución. Ingresó remitida para su valoración por el servicio de cirugía de pared abdominal, con clínica de dolor abdominal ocasional subcostal y estreñimiento. Ingresó con estudio tomográfico que reportó un defecto eventrógeno subcostal de $12 \times 15 \mathrm{~cm}$ con contenido hepático parcial, colon ascendente y epiplón.

Al examen físico se evidenció defecto de pared abdominal subcostal no reductible e incoercible cerca del reborde óseo costal compatible con eventración grande compleja con pérdida de domicilio (fig. 1).

Se propuso la realización de una eventroplastia con malla antes de la realización de neumoperitoneo preoperatorio progresivo hospitalario. El paciente aceptó y firmó el consentimiento informado. Se hospitalizó con un plan de neumoperitoneo preoperatorio progresivo. Se insufla día por medio con volúmenes de no más de 500 cc diarios, con un total de cuatro litros en diez días.

\section{Técnica quirúrgica}

Con anestesia general, se realizó la marcación del defecto, de la línea media y del reborde óseo. Se resecó la cicatriz previa mediante incisión losange, se disecó y se preservó el saco eventrógeno (fig. 2), que se dividió en una hoja superior y otra inferior, se realizó la reducción del contenido intestinal y adhesiólisis y se decidió, debido al tamaño del defecto y para movilizar tejidos hacia este, la separación de componentes anterior derecha, liberando la aponeurosis y el músculo oblicuo mayor (fig. 3). A continuación, se fijó la hoja superior del saco eventrógeno al borde inferior, dejando una porción o solapa por debajo del reborde costal (fig. 4), cerca del reborde costal con puntos en $U$, que son reparados y posteriormente anudados (fig. 5). Luego se fijó con sutura continua la hoja superior 
del saco eventrógeno a la aponeurosis sana cerca del borde inferior del defecto (fig. 5). Con un lavado previo con solución salina y control de hemostasia, se colocó una malla de polipropileno de $30 \times 30 \mathrm{~cm}$, fijada en sus bordes por punto simples cardinales, y en el medio, con dos líneas de sutura continua cerca de los bordes superior e inferior del defecto (fig. 5). Se dejó un drenaje a tejido celular subcutáneo, cierre de tejido celular subcutáneo y piel con nilón 2/0. La duración de la cirugía fue de dos horas.

El paciente evolucionó favorablemente en planta, con drenaje serohemático escaso, que se retiró al quinto día (fig. 6). Se le dio de alta con tolerancia de la vía oral y tránsito intestinal ese mismo día.

Acude a controles a los quince, treinta y noventa días y al año, con exploración física y ecográfica sin hallazgos de recidiva.

\section{DISCUSIÓN}

La reconstrucción de la pared abdominal es un desafío para el cirujano general y de pared abdominal. Debe tenerse en cuenta la calidad de las estructuras anatómicas afectadas ${ }^{8}$.

Cabe mencionar que no todos los servicios de pared abdominal cuentan con la disponibilidad de usar dos mallas en un paciente, y el acceso al adhesivo tisular es aún más difícil para realizar técnicas como la de Moreno Egea. Por esta razón, decidimos presentar la técnica con posicionamiento de las hojas de saco, colocando primero el inferior y dejando una porción del mismo o solapa debajo del reborde costal, tratando de simular la prótesis de refuerzo intraperitoneal retrocostal ${ }^{9}$. Lo ideal es restaurar la anatomía y la función de la pared abdominal utilizando la prótesis como refuerzo $0^{7,10}$. La técnica de doble malla, también llamada técnica de sándwich, la consideran LeBlanc y cols. en su metaanálisis, publicado en el año 2005 , como la técnica con menor porcentaje de recurrencia ${ }^{11}$, pero el acceso a estos elementos por parte de las empresas aseguradoras de salud en Argentina es limitado, razón por la que, a pasar de lo 
anteriormente mencionado, nos vimos en la obligación de diseñar una alternativa quirúrgica.

El método de adyuvancia preoperatoria escogido en este caso fue el uso del neumoperitoneo preoperatorio progresivo con el objetivo de aumentar el volumen de la cavidad abdominal entre un 35 y un $40 \%$, producir lisis de adherencias y reducir el volumen visceral. Asociado al uso de la separación de componentes técnica de Carbonell Bonafé Nivel uno, buscando un mayor avance de los tejidos y aumentar hasta en un $6 \%$ el volumen de la cavidad abdominal, para tratar de evitar el síndrome compartimental postoperatorio ${ }^{5}$. La reconstrucción fascial puede hacerse con separación de componentes musculoaponeuróticos de la vaina de los rectos abdominales y material protésico (sintético o biológico) ${ }^{8}$.

El uso de drenaje responde al proceso de facilitar la salida del líquido y de prevenir la aparición de complicaciones, aunque no existe consenso sobre su utilidad. Debe aclararse que su uso no influye en la aparición de complicaciones².

\section{CONCLUSIÓN}

Se presenta una alternativa para la reparación de la eventración subcostal compleja cuando no se dispone de dos mallas para la intervención plástica de la pared abdominal, con un buen resultado posoperatorio al seguimiento durante doce meses, dado que no todos los servicios ni sistemas de salud cuentan con recursos para emplear una técnica de doble malla.

\section{BIBLIOGRAFÍA}

1. González Sánchez JA, Valderrábano González S, Heras Garceau MA, Álvarez Peña E, Fernández Francos S. Nuestra experiencia en la técnica SAC de Carbonell-Bonafé en eventraciones complejas SAC. Rev Hispanoam Hernia. 2019;7(2):52-8. DOI: $\underline{10.20960 / \text { rhh. } 178}$ 
2. Torres Tejerizo JB, Bartoli JD, Dreizzen E. Utilidad del drenaje en las eventroplastias con malla. Rev Hispanoam Hernia. 2013;1(1):18-26. DOI: 10.20960/rhh.00277

3. Arias PM, Cervetti MR, Pasarín MA, Albornoz PD, Cacciavillani GC. Neumoperitoneo preoperatorio en eventración subcostal gigante. Rev Hispanoam Hernia. 2017;4(4). DOI: 10.20960/rhh.6

4. Peres MA de O, Aguiar HR, Andreollo NA. Surgical treatment of subcostal incisional hernia with polypropylene mesh-analysis of late results. Rev Col Bras Cir. 2014;41(2):82-6. DOI: 10.1590/S0100-69912014000200002

5. Mayagoitia JC, Armando H, Muñoz C. Guía de práctica clínica de la hernia incisional compleja. Rev Hispanoam Hernia 2021;9(2):95-104. DOI: 10.20960/rhh.00444

6. Derlin Juárez M. Manejo multimodal de la eventración gigante. Rev Hisp Hernia. 2018;6(1):3-10. DOI: 10.20960/rhh.57

7. Zorraquino González Á, Gutiérrez Ferreras Al, Elías Aldama M, García Carrillo M, Gómez Martínez JM, Taibo Asencor MÁ, et al. Reparación anatómica de la eventración subcostal Ángel. Rev Hispanoam Hernia 2015;3(4):161-7. DOI: 10.1016/j.rehah.2015.07.001

8. Palacios Juárez J, Morales Maza J, Rendón Medina MA, Ramírez Ramírez M, Rodríguez Quintero JH, Cortés Martínez LA. Reparación de defecto complejo de pared abdominal con colgajo una buena alternativa quirúrgica. Cir Cir. 2020;88(2):206-10. DOI: 10.24875/CIRU.19000671

9. Moreno-Egea A. La técnica de la doble reparación protésica (según Moreno-Egea) como solución en el caso de hernias subcostales masivas. Rev Hispanoam Hernia 2014;2(1):9-12. DOI: 10.1016/j.rehah.2013.08.004

10.Bellón JM, Moreno-egea A. Guía clínica para el uso de material protésico en la reparación de la hernia incisional. Rev Hispanoam Hernia 2021;9(2):137-42. DOI: 10.20960/rhh.00451 
11.Awad ZT, Puri V, LeBlanc K, Stoppa R, Fitzgibbons RJ, Iqbal A, et al. Mechanisms of ventral hernia recurrence after mesh repair and a new proposed classification. J Am Coll Surg. 2005;201(1):132-40. DOI: 10.1016/i.jamcollsurg.2005.02.035

\section{PIES DE LAS FIGURAS}

Figura 1. Paciente cuando ingresa en el Servicio de Cirugía General. Se observa defecto eventrógeno complejo subcostal derecho.

Figura 2. Disección de saco herniario antes de la liberación del neumoperitoneo.

Figura 3. Separación de componentes anterior. Se observa el músculo oblicuo liberado.

Figura 4. Gráfica que muestra la disposición de la hoja inferior del saco herniario en la reparación. Fuente: elaboración propia.

Figura 5. A. Avance de la hoja inferior del saco herniario al borde superior y subcostal con puntos en $U$ reparados con pinzas. B. Hoja inferior del saco eventrógeno fijada. C. Malla fijada con puntos separados cardinales y dos líneas de sutura continua mediales cerca de los rebordes del defecto.

Figura 6. La paciente cinco días después de la operación. Se observa drenaje serohemático. 